\title{
IDENTIFICATION AND MONITORING OF A PEM ELECTROLYSER BASED ON DYNAMICAL MODELLING
}

\author{
Lebbal, M.E. ${ }^{1}$ lebbal@ensm-douai.fr and Lecœuche, S. ${ }^{1,2}$ lecoeuche@ensm-douai.fr \\ ${ }^{1}$ Department A.I, Ecole des Mines de Douai, 941 rue Charles Bourseul, 59508 Douai Cedex France \\ ${ }^{2}$ Laboratoire LAGIS, USTL, Cité Scientifique-Bâtiment P2, 59655 Villeneuve d'Ascq Cedex France
}

\begin{abstract}
Hydrogen from water electrolysis associated with renewable energies is one of the most attractive solutions for the green energy storage. To improve the efficiency and the safety of such stations, some technological studies are still under investigation both on methods and materials. As methods, control, monitoring and diagnosis algorithms are relevant tools. These methods are efficient when they use an accurate mathematical model representing the real behaviour of hydrogen production system. This work focuses on the dynamical modelling and the monitoring of Proton Exchange Membrane (PEM) electrolyser. Our contribution consists in three parts: to develop an analytical dynamical PEM electrolyser model dedicated to the control and the monitoring; to identify the model parameters and to propose adequate monitoring tools. The proposed model is deduced from physical laws and electrochemical equations and consists in a steady-state electric model coupled with a dynamical thermal model. The estimation of the model parameters is achieved using identification and data fitting techniques based on experimental measurements. Taking into account the information given by the proposed analytical model and the experimentation data (temperature $T$, voltage $U$ and current $I$ ) given by a PEM electrolyser composed of seven cells, the model parameters are identified. After estimating the dynamical model, model based diagnosis approach is used in order to monitoring the PEM electrolyser and to ensure its safety. We illustrate how our algorithm can detect and isolate faults on actuators, on sensors or on electrolyser system.
\end{abstract}

\subsection{INTRODUCTION}

In recent decades, the global warming increases the average temperature of the air and oceans near the earth surface. This problem caused by $\mathrm{CO}_{2}$ gas and several polluting wastes continuing to affect the lives in the world. In order to overcome this problem, the use of renewable energy and its optimization become a humanity challenge [3]. An attractive solution is to integrate efficient energy storages. The hydrogen is one of most promising vectors to store green energy. In the last years, numerous stations including renewable energy and electrolyser have been developed in order to optimise the electric energy production by increasing the storage capacity. The key idea is to convert the hydrogen into electricity using Fuel Cell (FC) when the renewable energy is off (no wind, no sun). In order to have this fuel, during the high potential periods, the extra renewable energy is converted using a Proton Exchange Membrane (PEM) electrolyser into $H_{2}$. The global efficiency and safety of such installations (renewable energy source, fuel cell and electrolyser) lead to important research works in modelling, control [1] and monitoring. More precisely, it is necessary to propose an efficient supervision system. It permits to the user to decide if the hydrogen production station is faulty and if risk for itself and its environment could occur. For example, when sensor or actuator fault is detected, control laws could be adapted in order to put the system in fallback options. On the same way, maintenance could be planed in order to avoid major system failure (e.g. membrane breaking). In the aim to optimise control and monitoring of the hydrogen production and exploitation under safe conditions, the PEM cell modelling became very important and necessary.

This work is dedicated to the electrolyser safety for the development of monitoring and diagnosis tools. From the point of view our aim is to detect drifts that could lead to the critical failures by employing Fault Detection and Isolation (FDI) methods. Our contribution consists in three parts: modelling, identification and monitoring of the PEM electrolyser. 
According to the recent literature, the modelling task still needs improvements in order to develop models able to represent accurately the real dynamical equipment behaviour. Many electrolysis cell models have been proposed, these models are deduced from the thermal, the electrical and the fluid dynamics behaviours. Electrical model represents the relation between voltage $U$, current $I$ and temperature $T$ [7]. Thermal model represents the temperature $T$ variation and heat exchange [9]. The fluid dynamics model characterizes the chemical components movement, the pressure and the fluid volume [11]. Despite models variety, simplified electrical and thermal are mostly used [9]. Few advanced monitoring and control algorithms using a efficient modelling are really used in real conditions [10]. Our proposed model consists in a steady-state electrical model and a dynamic thermal model. These models are deduced from physical laws and electrochemical equations. The steady-state electric model represents algebra relations between voltage $U$, current $I$ and temperature $T$, it reflects steady behaviour. In order to reduce the model imprecision, these models need a parameters estimation using experimental data. This imprecision is a real limitation for the monitoring and diagnosis tools because the faults indicators and their sensitivity are strongly influenced by the parameters values. The identification of the electrical model parameters employs a non linear least square algorithm [5] and experimentation data (temperature $T$, voltage $U$ and current $I$ ) given by a PEM electrolyser composed of seven cells. The dynamic thermal model structure is inspired from the analytical heat transfer. Due to the fact that this model is linear and of order one. The identification of the thermal model parameters is developed using voltage $U$, current $I$, and temperature step responses.

Using these accurate models, it could be possible to develop efficient control system and on-line monitoring stage. In order to detect and isolate actuators, sensors or system faults, specific tools have to be designed [4]. For fault detection and isolation (FDI), we use model-based diagnosis approach. Based on the identified PEM electrolyser models, residuals are generated in order to check nonconsistency between the theoretical information and the measurements. These residuals contain only known or measured variables and are statistically nil in the absence of faults and different of zero when a fault occurs. In this paper we show modelling and identification results and illustrate that our monitoring algorithm (FDI) is able to detect and isolate faults.

The paper is organized in five sections. The first section concerns the introduction. The second section presents the PEM electrolyser principle. The third section exposes the electric and thermal behaviours and details our proposed models. The parameters estimation and the description of the used identification techniques are developed in fourth section. The monitoring approach is detailed and illustrates by results in the fifth part.

\subsection{THE PEM ELECTROLYZER PRINCIPLE}

The water electrolysis operation is an electrolytic process which decomposes water $\mathrm{H}_{2} \mathrm{O}$ molecule into oxygen $\mathrm{O}_{2}$ and hydrogen $\mathrm{H}_{2}$ gasses with the help of an electric current (figure 1). The electrodes submerged in an electrolyte (conductive medium) form the electrolysis cell. In PEM electrolysis cell, the electrolyte is a solid called membrane. In this latter, the $H^{+}$ions are used for electricity conductivity. We resume the water decomposition in followings Redox (Reduction and Oxidation) chemical reactions:

$2 \mathrm{H}_{2} \mathrm{O}+$ electric energy $\rightarrow 2 \mathrm{H}_{2}+\mathrm{O}_{2}$

- Oxidation Describes the electrons ( $\left.e^{-}\right)$loss (or the protons gain) by a molecule, atom or ion. In water electrolysis, at the anode electrode $(+)$ (oxygen production), the oxidation equation is:

$2 \mathrm{H}_{2} \mathrm{O} \rightarrow \mathrm{O}_{2}+4 \mathrm{H}^{+}+4 e^{-}$

- Reduction Describes the electrons ( $\left.e^{-}\right)$gain (or the protons loss) by a molecule, atom or ion. In water electrolysis, at the cathode electrode (-) (hydrogen production), the reduction equation is: 


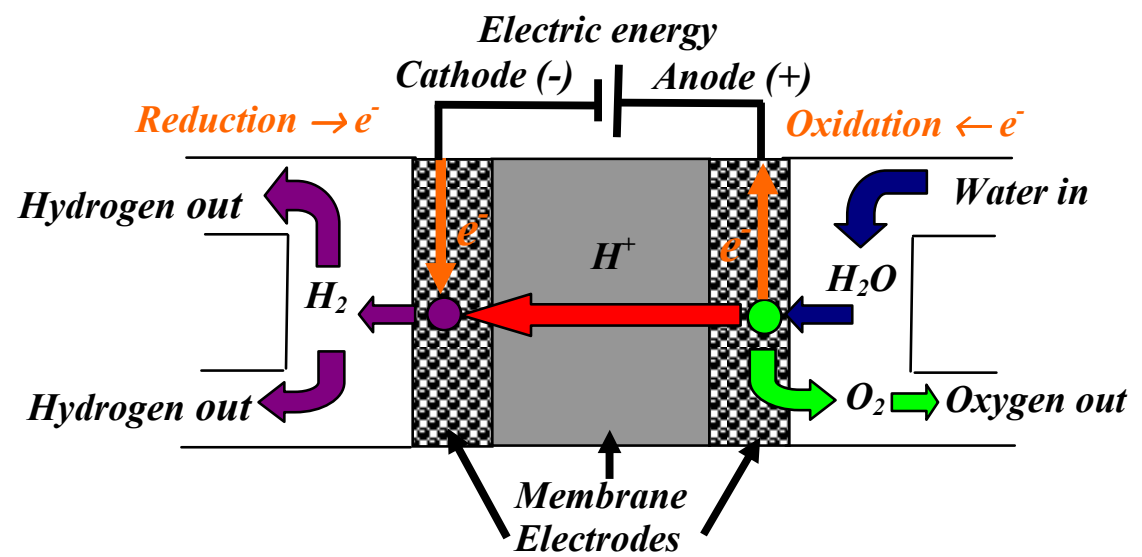

Figure 1: PEM electrolyser device

For splitting the water molecule, the electric energy is consumed by the Redox chemical reaction. As consequent, the change in enthalpy $\Delta H$, entropy $\Delta S$ (heat exchange=irreversible energy), and Gibbs energy (reversible energy) $\Delta G$ are closely related to the electric and the thermal energy generated by cell [9]. Assuming that the PEM electrolyser cell temperature $T[K]$ is given in Kelvin, for the water splitting, the change in enthalpy can be given according to the following equation:

$\Delta H=\Delta G+T \Delta S$

According to this equation, the electrical and thermal behaviours are combined. From the view of point of the electrolyser destruction, the current and the temperature are very significant. In one hand, high current could cause: electrode destruction, membrane melting, membrane drying or electrode pressure augmentation [8]. In other hand, a high temperature could induce: membrane hot point and membrane tear. In addition, these faults could cause a $\mathrm{H}_{2}$ (respectively $\mathrm{O}_{2}$ ) migration to $\mathrm{O}_{2}$ (respectively $\mathrm{H}_{2}$ ) side.

All these faults could be dangerous for the electrolyser and its environment. In order to detect, isolate and correct faults and then to improve the quality and the safety of the hydrogen production, this paper proposes a model-based diagnosis of a PEM electrolyser. This approach needs three processing steps : electrolyser modelling, parameters estimation and electrolyser monitoring which are detailed in the next sections.

\subsection{MODELLING APPROACH}

Using the energy change equation (4) and electrochemical process aspect, we can divide our electrolyser model into an electrical model completed with a thermal model. The electrical model is based on the energy exchange in link with the Gibbs energy and the electric source. The heat transfer equation is composed by the energy generated by entropy and the energy losses (or gained) by the external temperature and chemical components movement. In our proposed modelling approach, steady-state electrical model and dynamic thermal model are given based on energy conservation. The electrical model is deduced from an algebra relation between the current cell $I$ and voltage cell $U$ for a given temperature value $T$. The thermal model represents the dynamic temperature behaviours for both current $I$ and voltage $U$. The following figure illustrates our PEM electrolyser model.

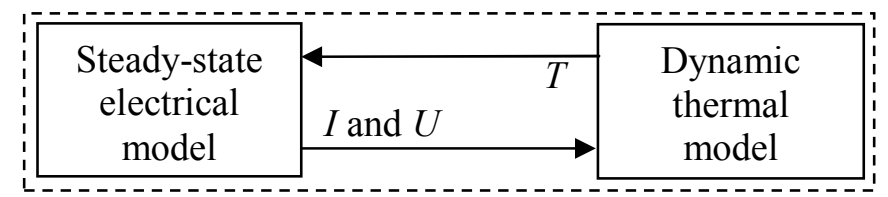


Figure 2:PEM electrolyser model

\subsection{Electrical model}

When the input voltage is applied to the PEM cell several voltage drops appear. These drops are characterized by an reversible drop $V_{\text {rev }}$, an activation drop $V_{a c t}$, an diffusion drop $V_{\text {diff }}$ and an ohmic losses $V_{\text {ohm }}$. The current circulating into the cell is influenced by these drops which are most of them non linear functions of the current. The electric schema is given in figure 3 .

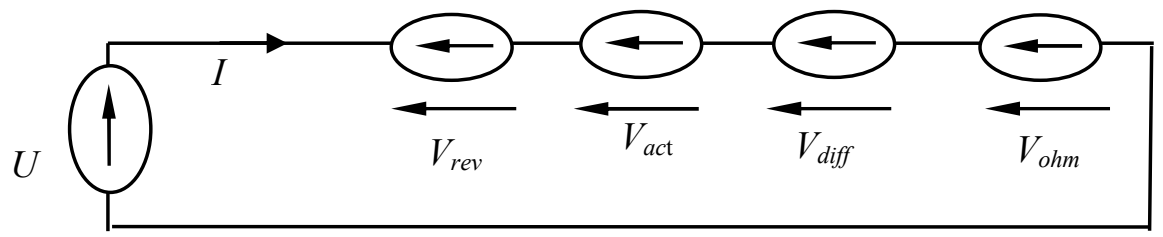

Figure 3 : Electric schema

From the figure 3, using circuit laws, we can write the following equation :

$U=V_{\text {rev }}+V_{\text {act }}+V_{\text {diff }}+V_{\text {ohm }}$

In order to build our steady-state electric model, we will study separately each voltage drop.

\subsubsection{Reversible voltage}

This voltage drop called sometimes open voltage is caused by the chemical Redox reaction. It represents the cell potential when the current is nil $(I=0)$. This reversible voltage $V_{\text {rev }}$ is calculated from the Gibbs energy, it can be defined by the Nernst equation [3] as follow:

$V_{\text {rev }}=V_{0}+\frac{R T}{2 F} \ln \left(\frac{P_{H 2} P_{O 2}^{I / 2}}{a_{H 2 O}}\right)$

Where $R=8,3144[\mathrm{~J} / \mathrm{mol} \mathrm{K}], F=96487$ [C $/ \mathrm{mol}], V_{0}=1.23[\mathrm{~V}], a_{H 2 O}=1$ (for liquid water), $P_{H 2}$ and $P_{\mathrm{O} 2}$ [atm] are universal gas constant, Faraday constant, standard reversible voltage, water activity the partially pressures of hydrogen and oxygen respectively.

\subsubsection{Activation voltage drop}

The activation voltage drop results from the proton transfer and chemical reaction velocity, it represents the electrochemical kinetic behaviours [2]. The activation voltage drop $V_{a c t}$ relation can be deduced from Butler-Volmer and Tafel laws [2]. Based on the work of Fonts [2], $V_{a c t}$ can be rewritten for an electrolyser, in function of the current $I$ as the following equation :

$V_{a c t}=\frac{R \cdot T}{\alpha \cdot n \cdot F} \ln \left(\frac{I}{I_{0}}\right)$

Where $\alpha, I_{0}$, are the transfer coefficient and the exchange current respectively.

\subsubsection{Diffusion voltage drop}

The fluids (gas and water) concentration and diffusion close to the electrodes influence the current values. Indeed diffusion behaviour causes variations of partial pressures and chemical reaction velocity. These changes induce voltage diffusion drop $V_{\text {diff. }}$ Based on an adaptation of diffusion equation proposed by Fonts [2] for a PEM fuel cell, the diffusion voltage $V_{\text {diff }}$ can be given by the following equation. 


$$
V_{\text {diff }}=\frac{R \cdot T}{\beta \cdot n \cdot F} \ln \left(1+\frac{I}{I_{\text {lim }}}\right)
$$

Where $\beta$ and $I_{\text {lim }}$ are the constant coefficient and the diffusion limit current respectively

\subsubsection{Ohmic voltage drop}

The resistor polymer membrane $R_{m e m}$ is the mainly cause of Ohmic voltage drop. The resistance value is often given by empirical relation (9) proposed by Springer and al [6]. As given in relation (9) $R_{m e m}$ is function of membrane section area $A_{m}\left[\mathrm{~cm}^{2}\right]$, membrane thickness $l_{m}[\mathrm{~cm}]$, hydration ratio $\lambda_{m}(=7$ dry enough $=14$ good hydration,$=22$ bathed) and temperature $T$.

$$
R_{\text {mem }}=\frac{l_{m}}{A_{m} \cdot\left(0.005139 \cdot \lambda_{m}+0.00326\right) \cdot \exp \left(1267\left(\frac{1}{303}-\frac{1}{T}\right)\right)}
$$

then the ohmic loss is given by:

$$
V_{\text {ohm }}=R_{m e m} \cdot I=\frac{l_{m}}{A_{m} \cdot\left(0.005139 \cdot \lambda_{m}+0.00326\right) \cdot \exp \left(1267\left(\frac{1}{303}-\frac{1}{T}\right)\right)} \cdot I
$$

\subsubsection{Electric model}

Substitute the voltage drops given by equations (6), (7), (8) and (10) in (5), we can deduced the steady state electrical model given by equation (11).

$U=V_{0}+\frac{R T}{2 F} \ln \left(\frac{P_{H_{2}} P_{O 2}^{I / 2}}{a_{\mathrm{H} 2 \mathrm{O}}}\right)+\frac{R \cdot T}{\alpha \cdot 2 \cdot F} \ln \left(\frac{I}{I_{0}}\right)+\frac{R \cdot T}{\beta \cdot n \cdot F} \ln \left(1+\frac{I}{I_{\text {lim }}}\right)+R_{\text {mem }} \cdot I$

The hydrogen flow is given in $[\mathrm{g} / \mathrm{s}]$ by :

$F_{H 2}=\frac{I}{2 F}$

\subsection{Thermal model}

The electrolysis cell temperature affects the relation between $U$ and $I$. In order to represent temperature behaviour and build a thermal model, we define all induced heat sources. We can consider four principal heat powers : chemical reaction (entropy), chemical components thermodynamic (gasses and water), external ambient temperature $T_{a}$ and joule effect caused by current circulation. In the aim to build the thermal model we use the heat energy conservation principle. For calculate chemical reaction heat energy, we use the thermoneutral cell voltage $V_{t h}=1.48[\mathrm{~V}]$ given by expression (13). This voltage remains almost constant or slightly changes with temperature. In addition we assume that the joule effect is neglected and the external temperature $T_{a}$ is constant. Then the thermal model can be written as the continuous dynamic equation (14):

$$
\begin{aligned}
& V_{t h}=\frac{\Delta H}{2 F} \\
& C_{p} \frac{d\left(T-T_{a}\right)}{d t}=\left(U-V_{t h}\right) \cdot I-h \cdot\left(T-T_{a}\right)
\end{aligned}
$$


Where $C_{p}[\mathrm{~J} / \mathrm{K}], h[\mathrm{~W} / \mathrm{K}], u=\left(U-V_{t h}\right) \cdot I$ and $h \cdot\left(T-T_{a}\right)$ are the electrolyser overall thermal capacity, the overall thermal admittance of electrolyser, the heat transfer rate generated by chemical reaction (caused by entropy energy) and the heat transfer rate caused by external temperature and fluids movement respectively.

Assuming that $u, x=\left(T-T_{a}\right)$ and $y=x$ are input, state and output of dynamic thermal model. The state and output equations in continuous case are given by the first order linear dynamic model (15):

$$
\left\{\begin{array}{l}
\frac{d x}{d t}=-\frac{h}{C_{p}} x+\frac{1}{C_{p}} u \\
y=x
\end{array}\right.
$$

Our modelling step allows having a PEM electrolyser model structure presented both by the steadystate electrical model (11) and the dynamic thermal model (15). We can note that several parameters $\left(\alpha, \beta, I_{0} I_{\text {lim }}, R_{\text {mem }}, C_{p}\right.$ and $h$, ) in the model are difficult to fix, because these values are specific to each electrolyser device. In order to use these models, their parameters have to be estimated using experimental data and identification techniques. In next part we propose and apply our identification approach for PEM electrolyzer.

\subsection{IDENTIFICATION APPROACH}

In this section, electrical and thermal models parameters are estimated using two distinct identification techniques. Electrical model parameters are estimated through a non-linear least square method [5] and thermal model parameters are identified using the properties of a first order linear model. Next sections present briefly these techniques and the estimated models.

\subsection{Electrical model identification}

\subsubsection{Non-linear least square identification principle}

We assume that we have $N$ measurements $\left(y_{k}, g_{k}(\theta, r)\right), k=0, . ., N$, as samples, $r$ represents the input and output regressor vector and $\theta \in I R^{p}$ is the parameter vector to be identified. Letting that a non linear relation $g_{k}$ between $y_{k}$ and $\theta$ at any time $k$ is given by :

$y_{k}=g_{k}(\theta, r)$

The parameter vector estimated by a non linear least square (NLS) is $\hat{\theta} \in I R^{p}$ which satisfy (17).

$G(\hat{\theta})^{T}(y-g(\hat{\theta}, r))=0$

Where, the observations vector $y \in I R^{N \times l}$ is composed by $y_{k}$, the regressor vector $g(\hat{\theta}, r) \in I R^{N \times l}$ contain observations function $g_{k}(\hat{\theta}, r)$ and the $G(\hat{\theta}, r)=\left[G_{k i}(\hat{\theta}, r)\right]$ with $G_{k i}=\frac{\partial g_{k}(\hat{\theta}, r)}{\partial \hat{\theta}_{i}}$. At the last of identification, we have $\hat{\theta} \rightarrow \theta(\hat{\theta}=\theta)$. For find $\hat{\theta}$ the optimal solution of (17), recursive algorithms can be used. We use in our case Gauss-Newton regression defined as:

$$
\hat{\theta}(j+1)=\hat{\theta}+\left.\left(G^{T}(\hat{\theta}, r) \cdot G(\hat{\theta}, r)\right)^{-1} \cdot G^{T}(\hat{\theta}, r) \cdot(y-g(\hat{\theta}, r))\right|_{\hat{\theta}=\hat{\theta}(j)}
$$

where $\hat{\theta}(j)$ and $\hat{\theta}(j+1)$ are the value of $\hat{\theta}$ at $j$ and $j+1$ iteration respectively. 


\subsubsection{Electrical model parameters identification}

We consider the static electrical model described by the equation (11). The basic idea is to fix the operating temperature $T=338[\mathrm{k}]$ and collect measurements $(U, I)$. From these measurements, the unknown parameters $\left(\alpha, \beta, I_{0} I_{\text {lim }}\right.$ and $\left.R_{m e m}\right)$ are identified applying the NLS method. The estimated parameters are defined by $\theta=\left[\begin{array}{lllll}\frac{1}{\alpha} & \frac{1}{I_{0}} & \frac{1}{\beta} & \frac{1}{I_{\text {lim }}} & R_{\text {mem }}\end{array}\right]^{T}$. Then the elements used for identification are defined as following

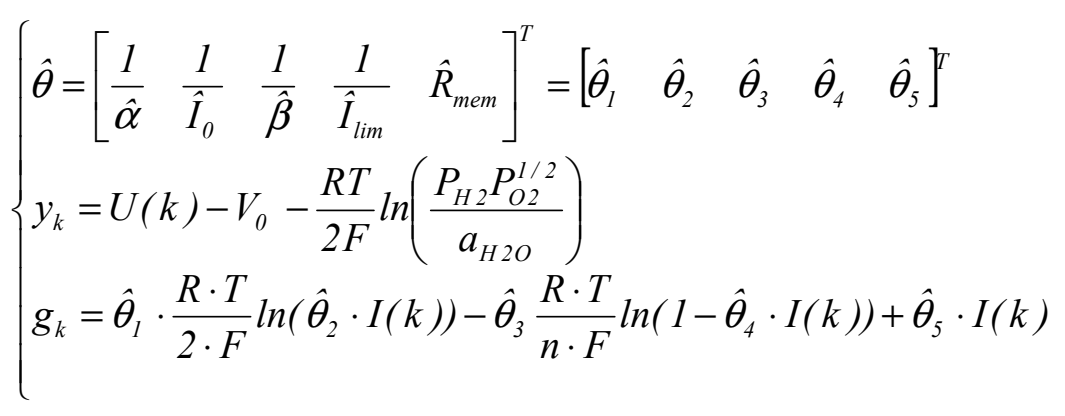
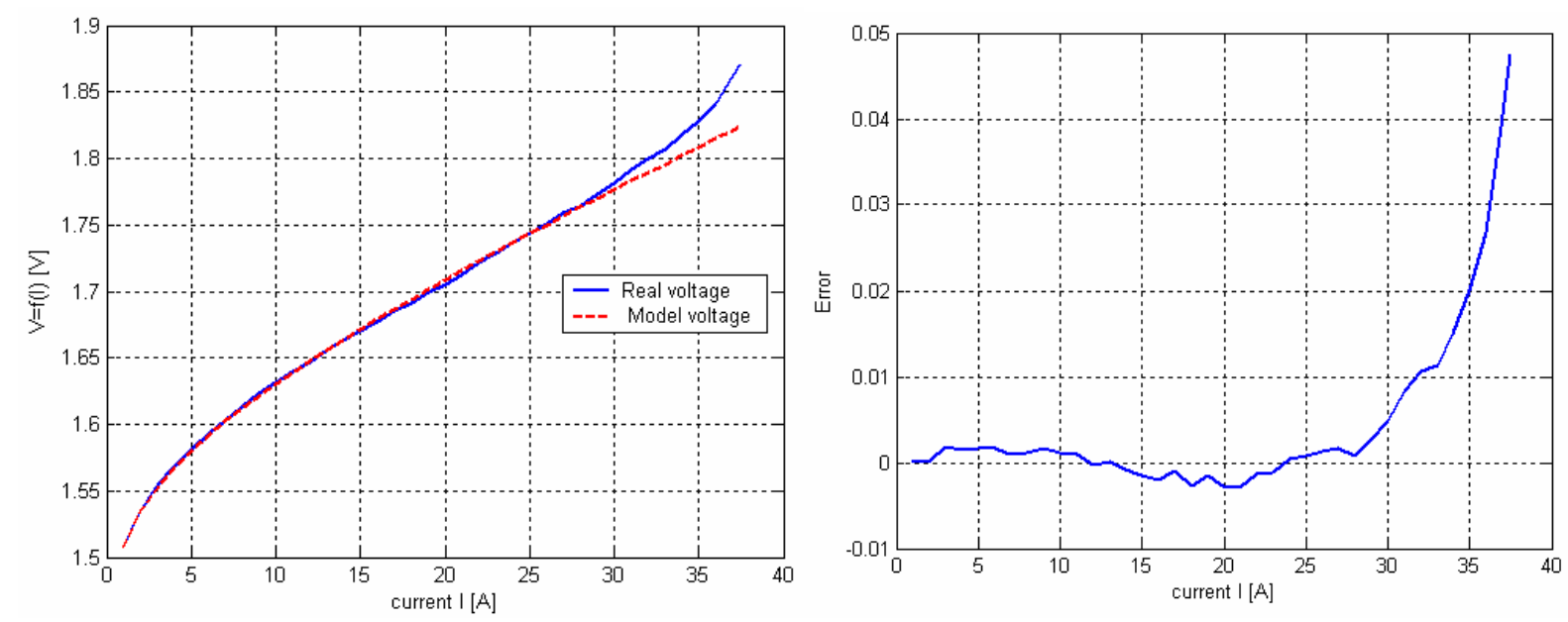

Figure 4: Real and identified electrical model

Applying the non linear least square method at real data given by the PEM electrolyser cell, we obtain the following parameters values: $\alpha=0.452 ; I_{0}=0.13 \cdot 10^{-3} ; \beta=0.04 ; I_{\text {lim }}=120 ;$ and $R_{\text {mem }}=3.2 \cdot 10^{-3}$. The figure 4 illustrates a comparison between the identified electrical model and the real device data. We note that our model only represents electric behaviours of PEM electrolyser. Indeed the error between our model and real data is very small, the average relative error equals to $0.32 \%$.

\subsection{Thermal model identification}

The parameters to be identified in the linear thermal model given by (15) are $C_{p}$ and $h$. From this model we can deduce the following Laplace transfer function $T P$ :

$$
T P=\frac{1 / h}{\left(\frac{C_{p}}{h} P+1\right)}
$$


The basic idea is to collect measurements $(U, I, T)$, then unknown $C_{p}$ and $h$ are identified using static gain $S_{g}$ and response time $t_{r}$ properties. Indeed in the first order linear dynamic system, $S_{g}$ and $t_{r}$ are related to $C_{p}$ and $h$ by following equation:

$$
\left\{\begin{array}{l}
S_{g}=\frac{1}{h}=\frac{\text { final value of } y}{\text { final value of } u}=\frac{\text { final value of }\left(T-T_{a}\right)}{\left(U-V_{t h}\right) \cdot I} \Rightarrow h=\frac{1}{S_{g}} \\
t_{r}=3 \cdot \frac{C_{p}}{h} \Rightarrow C_{p}=\frac{t_{r} \cdot h}{3}
\end{array}\right.
$$

Using this identification approach we have identified values $C_{p}=68544$ and $h=10.71$. The following figures illustrate the identified thermal model and real temperature data error for $(U=1.74, I=24)$ at external temperature $T_{a}=298[\mathrm{~K}]$. The identified thermal model is validated for a whole of admissible temperature variation $(283-333[\mathrm{~K}])$.
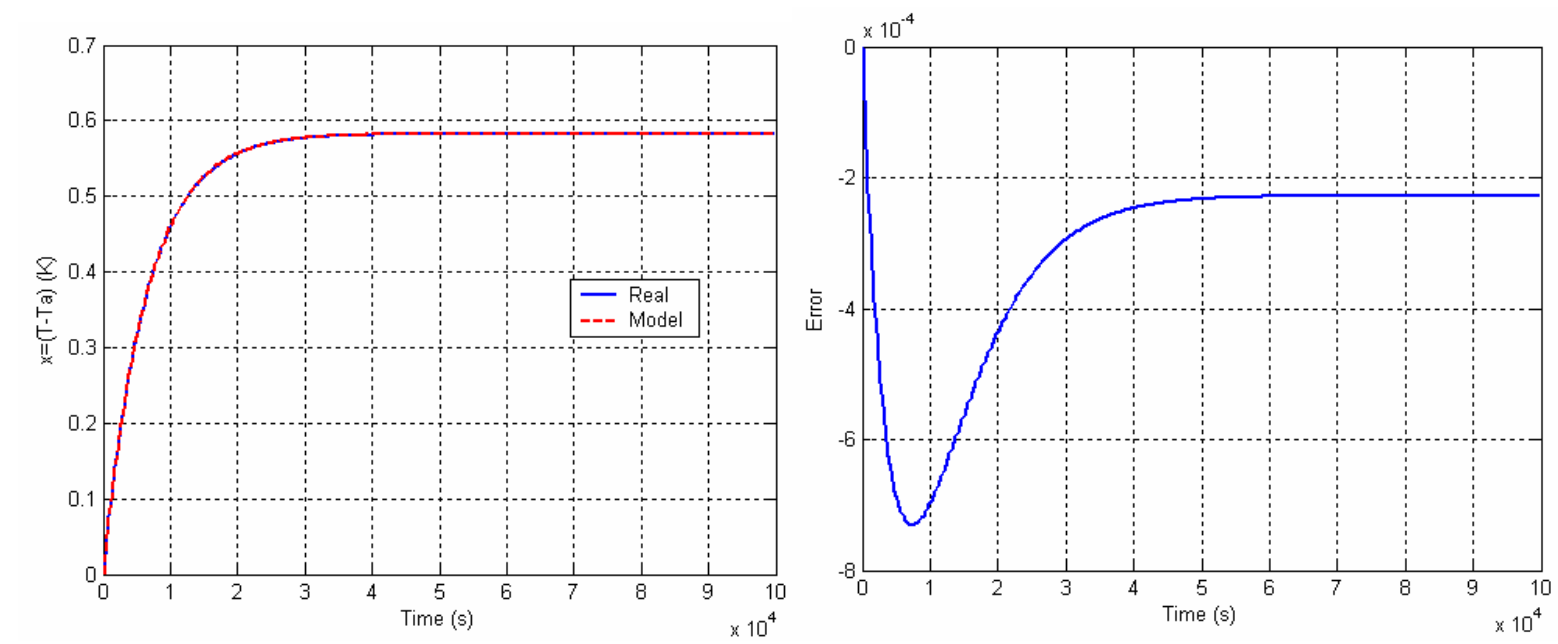

Figure 5: Real and identified thermal model for $U=1.74$ and $I=24$

As example from figure 5, we note that our thermal model represent accurately the temperature dynamic. Indeed the error between our model and real data is very small, the average relative error equals to $0.032 \%$.

Both the modelling and identification approach are developed in order to monitor and supervise a PEM electrolyser. In the next part our monitoring approach is proposed by introducing model based diagnosis algorithms.

\subsection{MONITORING APPROACH}

In order to improve the electrolyser safety, the monitoring is the supervision operation which permits to the user to analyse if the real system is (or would be) faulty or healthy (no-faults) [4]. In the aim to detect and isolate actuator, sensor or system faults, we use a model-based diagnosis approach. Based on our PEM electrolyser model, residuals are generated in order to check non-consistency between the theoretical information and the measurements. In an ideal situation, these residuals should remain zero in the healthy case and non-zero when a fault occurs. Among the methods used to generate residuals, the Analytical Redundancy Relations (ARR) permit to have equation where all the variables are known [4]. In other words, these relations are functions of only the measured inputs and outputs without requiring the use of the unmeasured states. In order to monitor the PEM electrolyser, the residuals are generated from electrical and thermal models using a parity space diagnosis approach [4]. Our monitoring structure (figure 6) and approach is described as follow. 


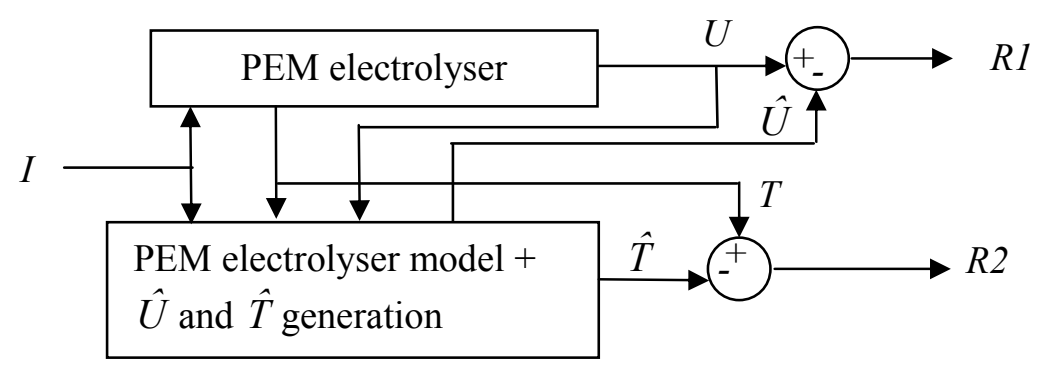

Figure 6: Monitoring approach

Using as measurement the voltage $U$, the current $I$ and the temperature $T$, two residuals $R I$ and $R 2$ are generated according to the PEM electrolyser model (11) and (15). The electrical model (11) can be induced the residual $R l$ at each sample time $k$ as :

$$
\left\{\begin{array}{l}
\hat{U}=V_{0}+\frac{R T(k)}{2 F} \ln \left(\frac{P_{H 2} P_{O 2}^{I / 2}}{a_{H 2 O}}\right)+\frac{R \cdot T(k)}{\alpha \cdot 2 \cdot F} \ln \left(\frac{I(k)}{I_{0}}\right)-\frac{R \cdot T(k)}{\beta \cdot n \cdot F} \ln \left(1-\frac{I(k)}{I_{\text {lim }}}\right)+R_{\text {mem }} \cdot I(k) \\
R I=U-\hat{U}
\end{array}\right.
$$

where $\left(\alpha, \beta, I_{0} I_{l i m}, R_{m e m}\right)$ are identified parameters

Using the thermal model (15) and the parity space diagnosis algorithm, the residual $R 2$ could be deduced. Indeed from (15) and (20) we can have linear discrete affined representation written as :

$$
y(k)=a \cdot y(k-1)+b \cdot u(k-1)=[y(k-1) \quad u(k-1)]\left[\begin{array}{l}
a \\
b
\end{array}\right]
$$

where in our case for a sample time $t_{e}=300 s \quad b=0.0043$ and $a=0.9542$.

then the residual $R 2$ can be given by the following equations:

$$
\left\{\begin{array}{l}
\hat{T}(k)=a \cdot\left(T(k-1)-T_{a}\right)+b \cdot\left(U(k-1)-V_{t h}\right) \cdot I(k-1)+T_{a} \\
R 2=T(k)-\hat{T}(k)
\end{array}\right.
$$

We use model parameters $\left(\alpha, \beta, I_{0} I_{\text {lim }}, R_{\text {mem }}, a, b\right)$ estimated in the section 4 and measurement $(U(k), I(k), T(k), T(k-1))$ to calculate instantaneous values of residuals $R I\left(U, I, T, \alpha, \beta, I_{0} I_{\text {lim }}, R_{\text {mem }}\right)$ and $R 2(U, I, T, a, b)$. When residuals are nil, it could be estimated that the actuators, the sensors and the PEM electrolyser system are healthy. In the opposite, non zero residuals imply fault presences. The fault detection and isolate could be diagnosed using a simple thresholding logic. A test is achieved on the residual by comparison with a threshold $S$ fixed according to the measurement noise and the modelling uncertainties. A basic fault isolation decision is given in the table 1.

Table 1: Faults isolation

\begin{tabular}{|l|c|c|c|c|}
\hline & $\begin{array}{c}\text { Non fault } \\
\text { System is healthy }\end{array}$ & $\begin{array}{c}\text { Thermal model } \\
\text { is faulty }\end{array}$ & $\begin{array}{c}\text { electrical model } \\
\text { is faulty }\end{array}$ & $\begin{array}{c}\text { Sensors or actuators are } \\
\text { faulty }\end{array}$ \\
\hline$R 1$ & $|R 1|<S$ & $|R I|<S$ & $|R 1|>S$ & $|R 1|>S$ \\
\hline$R 2$ & $|R 2|<S$ & $|R 2|>S$ & $|R 2|<S$ & $|R 2|>S$ \\
\hline
\end{tabular}


In the following figures we illustrate our simulation for healthy and faulty cases. The results are given for current input $I=2$ [A], with electrodes pressure equal to 1 [atm]. The faults occur at time $12000 \mathrm{~s}$ and vanish at time 60000s.

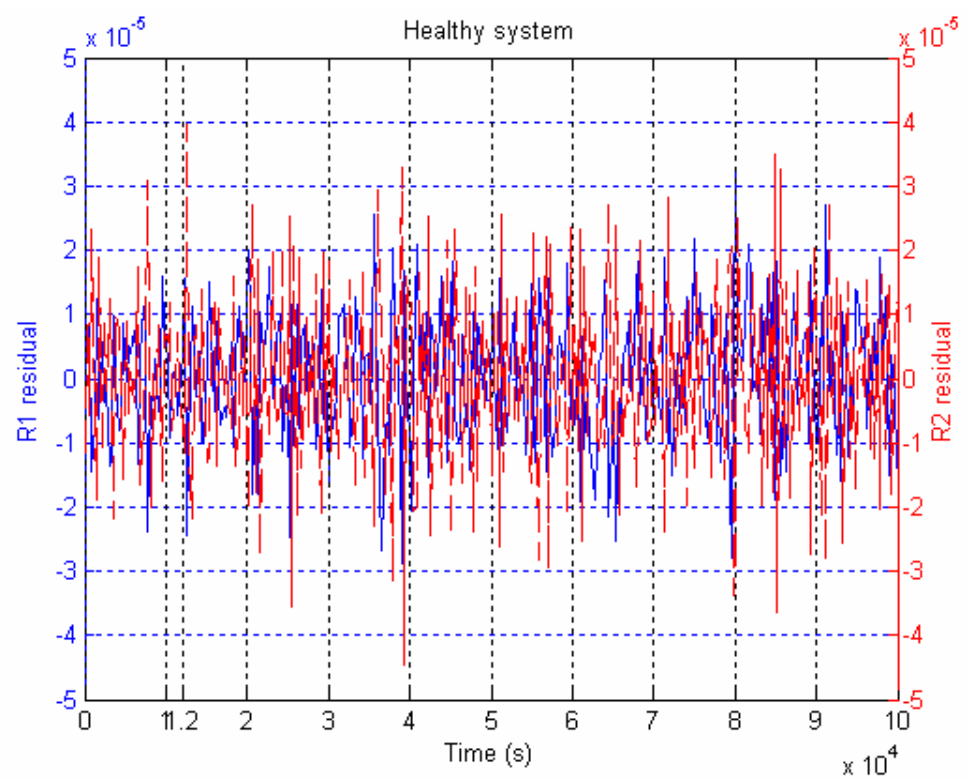

Figure 7: Residuals $R 1$ and $R 2$ in healthy case

From figure 7, corresponding to the healthy behaviours we note that the residuals $R 1$ and $R 2$ are both close to zero.

To illustrate our monitoring algorithm efficiency, we represent in the following figures, the residuals ( $R 1$ and $R 2$ ) evolutions when three faults occur. Figure 8 shows the behaviours when the desired current actuator value is deviated by a fault equal to $0.3 \mathrm{~A}$. Figure 9 illustrates the residuals behaviours when the overall thermal admittance $h$ parameter value is deviated. It takes a value equal to $(h+90)$ corresponding to a heat exchange fault. In figure 10, residuals evolutions due to a membrane fault are represented. In this case, the membrane resistor deviation equals to $3 \Omega\left(R_{m e m}+3\right)$.

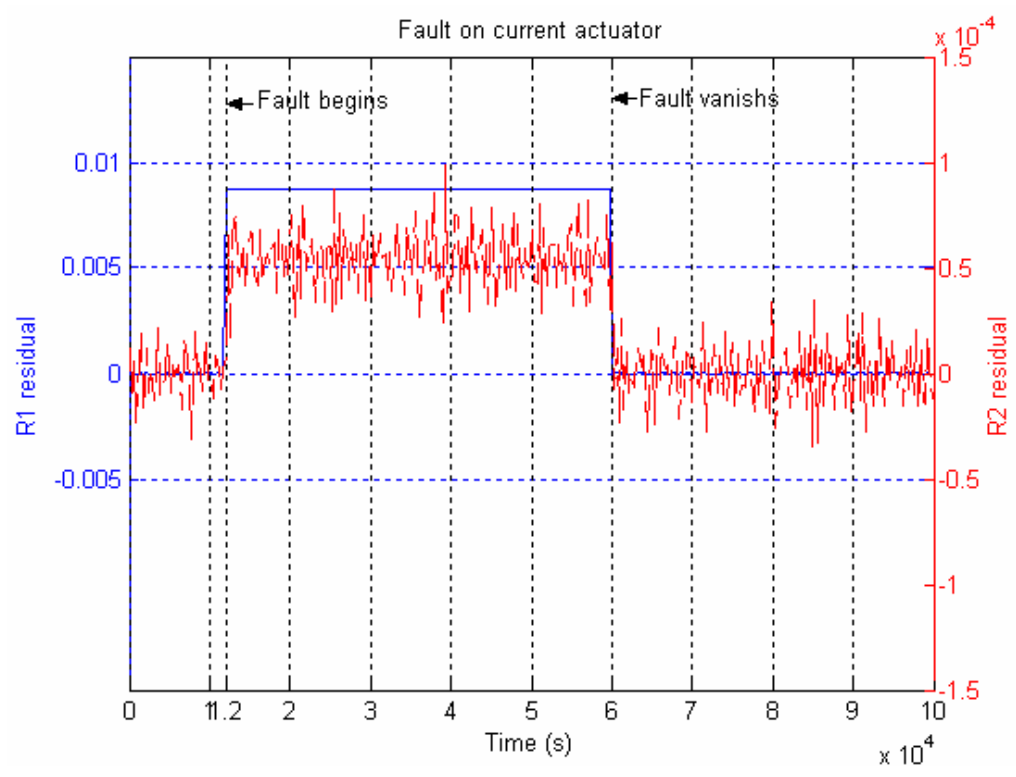

Figure 8: Residuals $R 1$ and $R 2$ in case of an actuator fault 


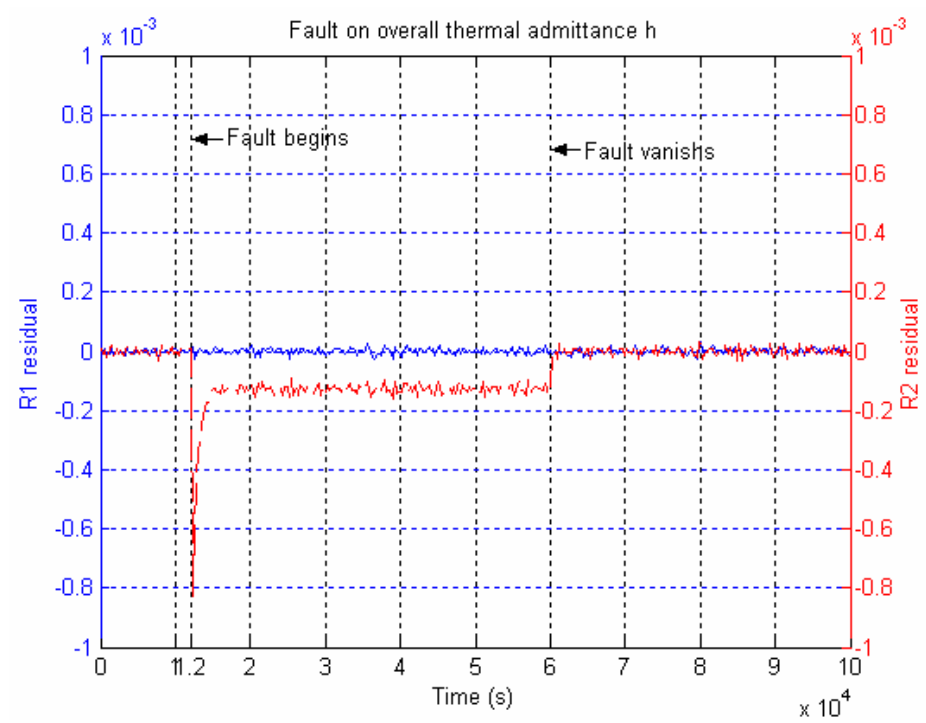

Figure 9: Residuals $R 1$ and $R 2$ when one thermal model parameter is faulty

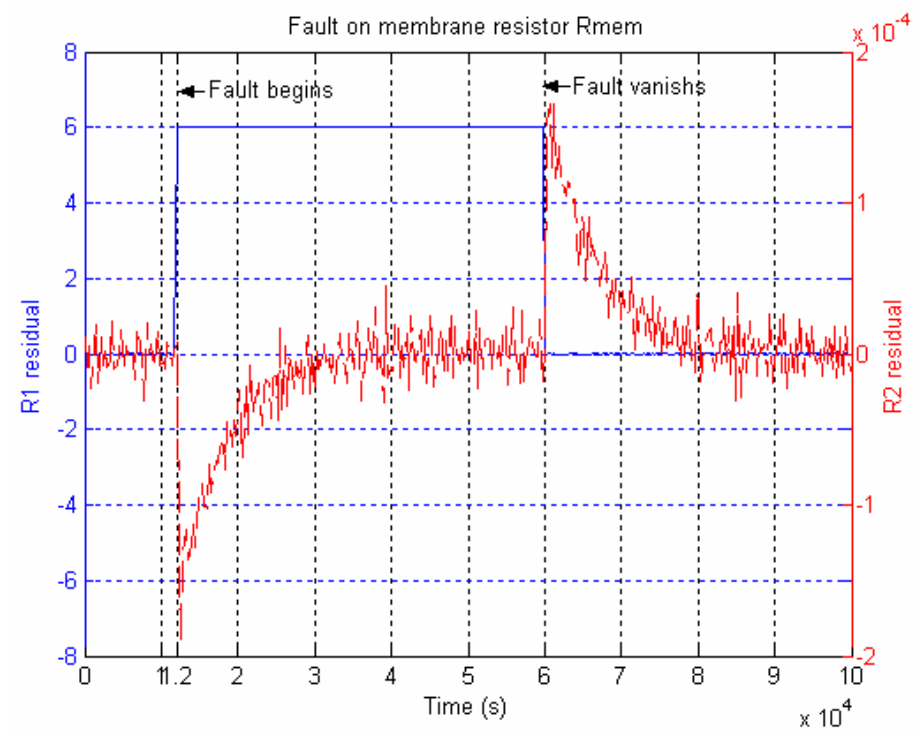

Figure 10: Residuals $R 1$ and $R 2$ when one electrical model parameter is faulty

In figure 8,9 and 10, we remark that for the two residuals $R 1$ and $R 2$, the choice of two threshold permit the fault detection. The optimal threshold values are defined in order to increase the fault detection and decrease the false alarm. In figure 8 and 9, we note that the sensor and thermal model fault can be isolated according to the table 1 . But in figure 10 the electrical model fault can be isolated and confirmed after a delay corresponding to the residual $R 2$ evolution. This delay is due to the thermal model response time seeing that the electrical model and thermal are closely coupled. Indeed in this case electrical model is influenced by temperature and thermal model is influenced by the faulty voltage sensor. Our monitoring results illustrate that we can avoid several critical and dangerous cases as presented in $\S 2$. Indeed the current actuator monitoring permits to avoid electrode destruction, membrane melting, membrane drying or electrode pressure augmentation [8]. The temperature sensor monitoring allows avoiding membrane hot point, membrane tear. In this sense, the membrane monitoring is an important tool to ensure the electrolyser safety. 


\subsection{CONCLUSIONS}

In this work we have developed for PEM electrolyser a model, an identification approach and a diagnosis algorithm. Our model consists in steady-state electrical model and linear dynamic thermal model. The PEM electrolyser model is validated with an identification approach through real experiments. The identification algorithm uses a non linear least square method for electrical model parameters estimation and the thermal model parameters are estimated using the first order response properties. In order to have a safe electrolyser operating mode, our identified model is used for developing monitoring algorithms. Our monitoring approach is based on a model based diagnosis method, it permits the detection and the isolation of sensors, actuators and (or) system faults. Our diagnosis approach can be used to avoid electrode destruction, membrane melting, membrane drying, electrode pressure augmentation, membrane hot point and membrane tear. Several results are presented in order to illustrate the advantage of our modelling, identifying and monitoring methods. The perspective of this work is to improve monitoring approach by the use of adaptive threshold detection and the study fault detectors (residuals) sensitivity to a several PEM electrolyser parameters.

\section{REFERENCES}

1. Chiu, L.Y, Diong, B and Gemmen, R.S. An improved small-signal model of the dynamic behavior of pem fuel cells. IEEE transactions on industry applications, vol. 40, no. 4, pp970-977, july/august 2004.

2. Fontes, G. Modélisation et caractérisation de la pile PEM pour l'étude des interactions avec les convertisseurs statiques. Thèse doctorat, laboratoire d'électrotechnique et d'électronique industrielle de l'ENSEEIHT UMR CNRS, l'institut national polytechnique de Toulouse, 16 septembre 2005.

3. Francois, B, Hissel, D and Iqbal, M.T. Dynamic modelling of a fuel cell and wind turbine DClinked power system Electrimacs conference, Hammamet, Tunisia, April 17-20 2005

4. Lebbal. M. Contribution à la modélisation et au diagnosis des systèmes à commutations. PHD thèse de l'université du havre, Le Havre, France 2006.

5. Ljung,L. System identification: Theory for the user. Prentice-Hall information and system sciences series, Prentice-Hall, Englewood Cliffs, New Jersey, 1999

6. Springer, T. E, Zawodinski, T. A and Gottesfeld, S. Polymer electrolyte fuel cell model, Journal of the Electrochemical Society, vol. 138, no 8, pp. 2334-2342,1991.

7. Wang, C, Nehrir, M.H and Shaw, S.R. Dynamic models and model validation for PEM fuel cells using electrical circuits. IEEE transactions on energy conversion, vol. 20, no. 2, pp 442 452, June 2005.

8. Tekin, M, Hissel, D, Pera, M and Kauffmann, J. Energy consumption reduction of a PEM fuel cell motor-compressor group thanks to efficient control laws. Journal of Power Sources 156, pp 57-63 (2006).

9. Ulleberg, O. Modeling of advanced alkaline electrolyzers: a system simulation approach. International Journal of Hydrogen Energy 28, pp21-33 , 2003.

10. Vosen, S.R. Keller, J.O. Hybrid energy storage systems for stand-alone electric power systems: optimization of system performance and cost through control strategies International Journal of Hydrogen Energy 24, pp1139-1156, 1999.

11. Yu, Q, Srivastava, A.K, Choe, S.Y and Gao, W. Improved modeling and control of a PEM fuel cell power system for vehicles". Proceedings of the IEEE Southeast Conference, pp 331- 336 , March 31 - April 2, 2006. 